\title{
Single top quark production in association with a W boson in CMS
}

\author{
Víctor Rodríguez Bouza on behalf of the CMS Collaboration*† \\ Universidad de Oviedo, Instituto Universitario de Ciencias y Tecnologías Espaciales de Asturias \\ (ICTEA), Spain \\ E-mail: victor.rodriguez.bouza@cern.ch
}

\begin{abstract}
The production of a top quark in association with a $\mathrm{W}$ boson $(\mathrm{tW})$ is one of the single top processes that takes place at LHC. The interest of this production relies on the quantum interference at NLO with the top-antitop pair production one of the most relevant processes in the LHC. The measurements of the tW production cross section using data collected by CMS at $13 \mathrm{TeV}$ are presented. The results are confronted with precise theory calculations.
\end{abstract}

European Physical Society Conference on High Energy Physics - EPS-HEP2019 -

10-17 July, 2019

Ghent, Belgium

\footnotetext{
* Speaker.

†Partially funded by "Ayudas para contratos predoctorales para la formación de doctores" (FPI) program of the Ministerio de Ciencia, Innovación y Universidades (Gobierno de España).
} 


\section{Introduction}

The top quark is a very relevant fermion inside the standard model (SM) due to its high mass, thanks to which it is the particle that most interacts with the Higgs boson. Consequently, it plays an important role in stabilising the Higgs field. There are various ways of producing top quarks. The set of processes where the top quark is not produced in top-antitop pairs is called single top, and their main interest is that they display at least one $\mathrm{Wtb}$ vertex. This electroweak interaction allows to probe the $\mathrm{V}_{\mathrm{tb}}$ element of the CKM matrix. The difference between these processes (or channels) is the virtuality of the W boson: if it is produced (on-shell) in the final state, the process is called $\mathrm{tW}$ (see Fig. 1). If the top quark is produced through the exchange of a virtual $\mathrm{W}$ boson, then we have the $\mathrm{t}$ channel. And, if the top quark arises in the final state due to the decay of a $\mathrm{W}$ boson, then is called s channel.
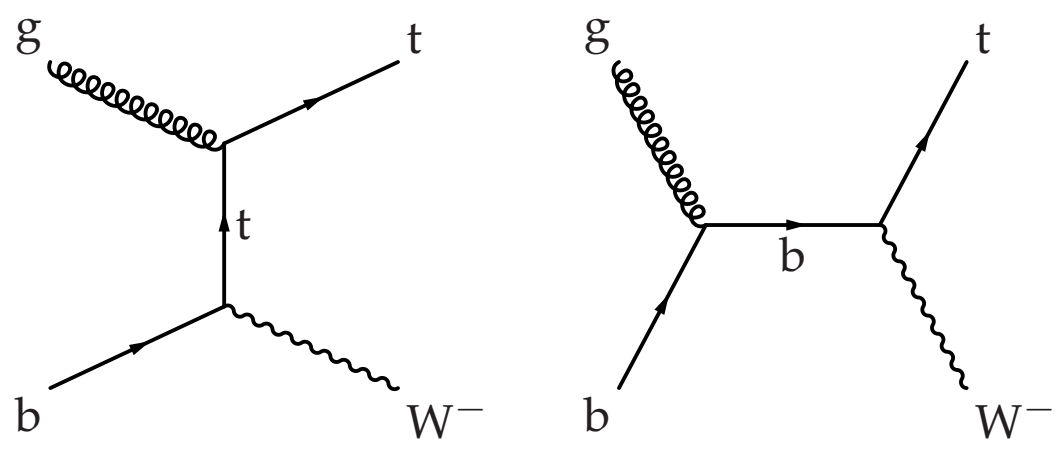

Figure 1: Leading order Feynman diagrams for single top quark production in the tW mode, the chargeconjugate modes are implicitly included [1].

Both CMS [2] and ATLAS [3] have observed this process at $\sqrt{s}=8 \mathrm{TeV}$, and CMS also at $\sqrt{s}=13 \mathrm{TeV}$ [1], with higher precision due to the increase of the cross section of the process as well as the increase of integrated luminosity. This last analysis is the one detailed in this document.

\section{Methodology}

In order to maximise the signal presence in our selection, we choose leptonic events with one electron and one muon, of opposite charge. This way we look after the decay of both $\mathrm{W}$ bosons of the process (as the top quark almost always decays to a $\mathrm{W}$ boson and a bottom quark). In addition, the most energetic lepton in the transverse plane must have $\mathrm{p}_{\mathrm{T}}>25 \mathrm{GeV}$ and the other $\mathrm{p}_{\mathrm{T}}>20$ $\mathrm{GeV}$. The trigger selection to choose them is a combination of di-lepton and single-lepton ones.

At this point, the classification depending on the number of jets and $b$ jets in each event is a nice way to further reduce the background contribution, which is not at all negligible, as seen in Fig. 2. We focus on those events that present a jet that must also be tagged as coming from a bottom quark (1j1b). In addition, we consider two more categories: that with two jets and one b-tagged jet $(2 j 1 b)$ and the one with two jets that are $b$-tagged $(2 j 2 b)$.

To optimise the signal extraction and to improve the discrimination between tW events and $\mathrm{t} \overline{\mathrm{t}}$ background, we have used a multivariate approach, building two boosted decision trees (BDT) trained in the $1 \mathrm{j} 1 \mathrm{~b}$ and $2 \mathrm{j} 1 \mathrm{~b}$ regions. The first one is designed to exploit that $\mathrm{t} \overline{\mathrm{t}}$ events in the $1 \mathrm{j} 1 \mathrm{~b}$ 


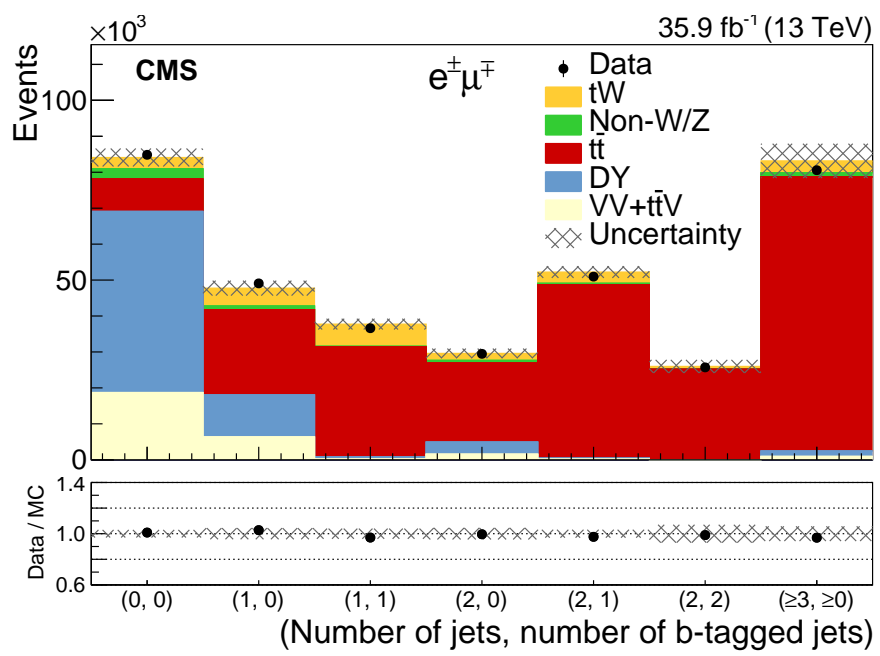

Figure 2: Yields observed in data, compared with those expected from simulation, as a function of the number of jets and number of b-tagged jets for events passing the baseline dilepton selection. The error band includes the statistical and all systematic uncertainties, except those from background normalisation. The bottom of each panel shows the ratios of data to the sum of the expected yields. [1]

region are mostly present due to a jet that lies out of the acceptance. Thus, the number of loose jets (those with $\mathrm{p}_{\mathrm{T}}>20 \mathrm{GeV}$ ) or the boost of the system conformed of the two leptons, the jet and the $\mathrm{p}_{\mathrm{T}}^{\mathrm{miss}}$ are relevant variables for it. In the case of the $2 \mathrm{j} 2 \mathrm{~b}$ region BDT, angular correlations between the jets, $\mathrm{p}_{\mathrm{T}}^{\text {miss }}$ and the leptons are the most discriminating distributions.

With this selection, we perform a maximum likelihood fit to obtain the signal contribution. To do so, the signal strength is fitted at the same time as different nuisance parameters that model the effect of the various uncertainties. The fit is done over the $1 \mathrm{j} 1 \mathrm{~b}$ BDT discriminant and the $2 \mathrm{j} 1 \mathrm{~b}$ BDT discriminant distributions as well as over the subleading jet $\mathrm{p}_{\mathrm{T}}$ distribution in the $2 \mathrm{j} 2 \mathrm{~b}$ region (see Fig. 3). To further enhance the discrimination of the BDT output distributions, the binning is chosen so that the contribution of $t \bar{t}$ is constant in all of them. Then, for the three variables, the information of each bin is modelled in the likelihood as Poisson density functions and the influence of uncertainties (i.e. the nuisance parameters) is taken into account through log-normal products.

The procedure to extract the $68 \%$ confidence interval is described in [4], done by modifying the test statistic by one unit from the minimum value found in the fit. Statistical and systematic uncertainties are taken into account by fixing all nuisance parameters to their post-fit value in the first case and by fixing all except the one of the chosen uncertainty to assess in the second one. In Tab. 1 the final uncertainties (in percentage) of the different systematic and statistical sources are shown, as well as the global amount (obtained by quadratic sum of the previous ones).

\section{Results and discussion}

Once the fit is performed, the tW inclusive cross section measurement is derived from the obtained signal strength, and it is found to be $63.1 \pm 1.8$ (stat.) \pm 6.4 (syst.) \pm 2.1 (lumi.) pb. As seen in Tab. 1, the global amount of uncertainty is $11 \%$, which is mainly driven by the contribution of luminosity, trigger and lepton efficiency uncertainty sources: dominant in all the bins of the fit. 

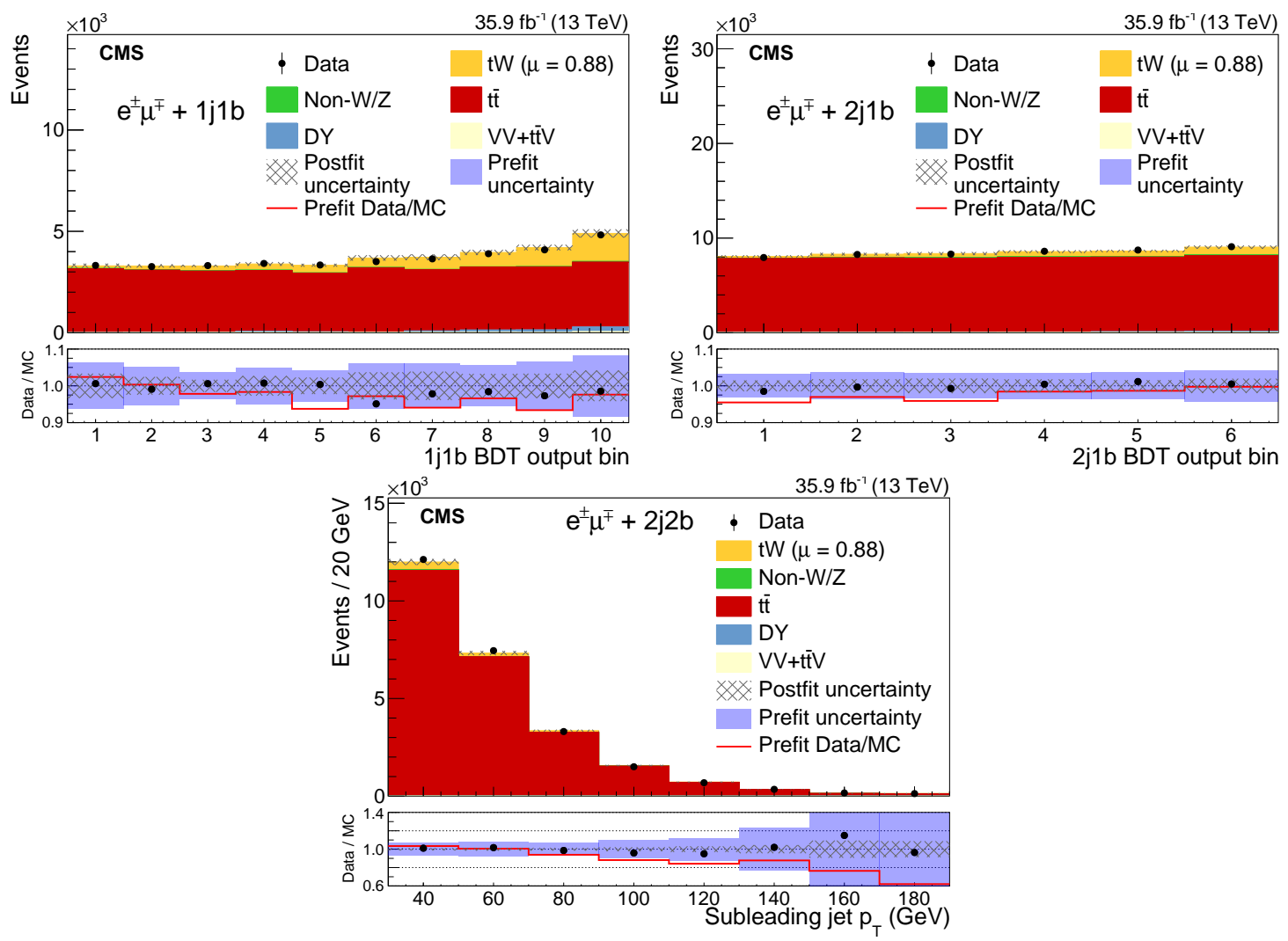

Figure 3: Comparison of the BDT output in the $1 \mathrm{j} 1 \mathrm{~b}$ (upper left) and $2 \mathrm{j} 1 \mathrm{~b}$ (upper right) regions and the $\mathrm{p}_{\mathrm{T}}$ of the subleading jet in the $2 \mathrm{j} 2 \mathrm{~b}$ region (lower) distributions after the fit is performed for the observed data and simulated events. The error band includes the statistical and systematic uncertainties. The bottom of each panel shows the ratios of data to the prediction from simulations (line) and from the fit (dots), together with their corresponding uncertainties (solid and hatched band, respectively) [1].

The measurement is in agreement with the SM predicted value at next-to-leading order, which is $71.7 \pm 1.8$ (scale) $\pm 3.4\left(\mathrm{PDF}, \alpha_{\mathrm{S}}\right) \mathrm{pb}[5]$.

\section{References}

[1] CMS Collaboration, "Measurement of the production cross section for single top quarks in association with $\mathrm{W}$ bosons in proton-proton collisions at $\sqrt{s}=13 \mathrm{TeV}$ ", JHEP 10 (2018) 117.

[2] CMS Collaboration, "Observation of the associated production of a single top quark a W boson in pp collision at $\sqrt{s}=8$ TeV." Phys. Rev. Lett. 112 (2014) 231802.

[3] ATLAS Collaboration, "Measurement of the production cross section of a single top quark in association with a W boson at $8 \mathrm{TeV}$ with the ATLAS experiment", JHEP 01 (2016) 064.

[4] CMS Collaboration, "Precise determination of the mass of the Higgs boson and tests of compatibility of its couplings with the standard model predictions using proton collisions at 7 and $8 \mathrm{TeV}$ ", Eur. Phys. J. C 75 (2015) 212.

[5] N. Kidonakis, "Theoretical results for electroweak-boson and single-top production", in Proceedings, 23rd International Workshop on Deep-Inelastic Scattering and Related Subjects (DIS 2015): Dallas, Texas, USA, April 27-May 01, 2015, volume DIS2015, p. 170. 2015. arXiv:1506.04072. 


\begin{tabular}{|c|c|}
\hline Source & Uncertainty (\%) \\
\hline \multicolumn{2}{|l|}{ Experimental } \\
\hline Trigger efficiencies & 2.7 \\
\hline Electron efficiencies & 3.2 \\
\hline Muon efficiencies & 3.1 \\
\hline JES & 3.2 \\
\hline Jet energy resolution & 1.8 \\
\hline b tagging efficiency & 1.4 \\
\hline Mistag rate & 0.2 \\
\hline Pileup & 3.3 \\
\hline \multicolumn{2}{|l|}{ Modeling } \\
\hline$\overline{\mathrm{t}} \overline{\mathrm{t}} \mu_{\mathrm{R}}$ and $\mu_{\mathrm{F}}$ scales & 2.5 \\
\hline $\mathrm{tW} \mu_{\mathrm{R}}$ and $\mu_{\mathrm{F}}$ scales & 0.9 \\
\hline Underlying event & 0.4 \\
\hline Matrix element/PS matching & 1.8 \\
\hline Initial-state radiation & 0.8 \\
\hline Final-state radiation & 0.8 \\
\hline Color reconnection & 2.0 \\
\hline B fragmentation & 1.9 \\
\hline Semileptonic B decay & 1.5 \\
\hline PDFs & 1.5 \\
\hline DR-DS & 1.3 \\
\hline \multicolumn{2}{|l|}{ Background normalization } \\
\hline$\overline{\mathrm{t}} \overline{\mathrm{t}}$ & 2.8 \\
\hline VV & 0.4 \\
\hline Drell-Yan & 1.1 \\
\hline Non-W / Z leptons & 1.6 \\
\hline $\mathrm{t} \overline{\mathrm{t}} \mathrm{V}$ & 0.1 \\
\hline MC finite sample size & 1.6 \\
\hline Full phase space extrapolation & 2.9 \\
\hline $\begin{array}{l}\text { Total systematic } \\
\text { (excluding integrated luminosity) }\end{array}$ & 10.1 \\
\hline Integrated luminosity & 3.3 \\
\hline Statistical & 2.8 \\
\hline Total & 11.1 \\
\hline
\end{tabular}

Table 1: Estimation of the effect on the signal strength of each source of uncertainty in the fit. Experimental and modeling uncertainties affect both the rate and the shape of the templates while background normalisation uncertainties affect only the rate. [1]. 